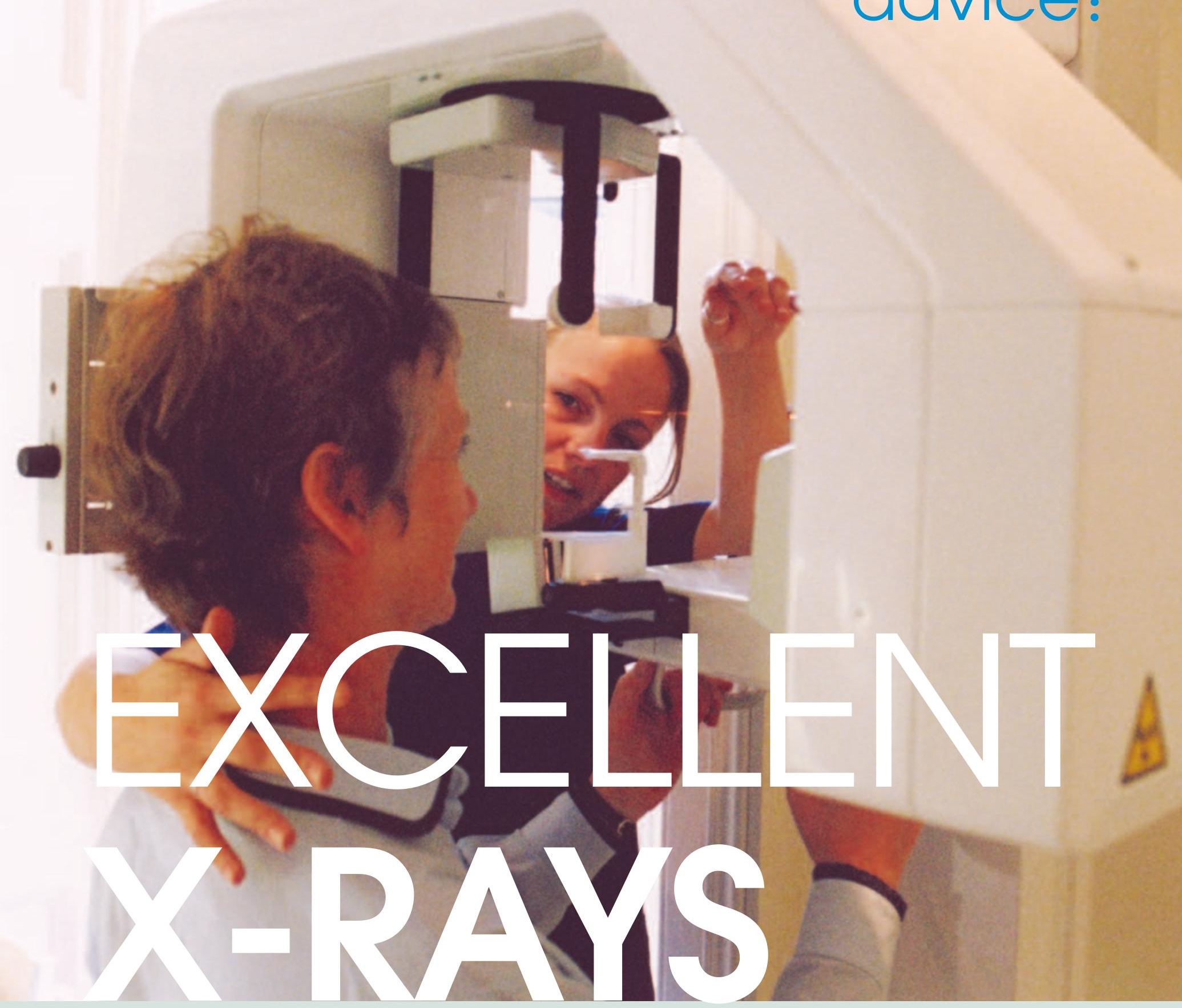

Naomi Scott gives

an overview of

radiography in the dental practice.

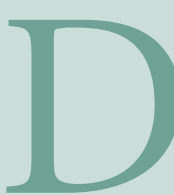

ental $\mathrm{x}$-rays are the most frequently taken $\mathrm{x}$-rays in the whole of the UK. It is important to patients, the staff taking the $\mathrm{x}$-ray and the rest of the staff at the practice that this is done in the safest and most effective ways possible. Everybody has a role to play and although the risks involved in ionising radiation are substantial, they can be kept to a minimum with proper training, good practice and quality control. This article is a brief reminder for those who are qualified in dental radiography and an introduction to those who are interested in increasing their responsibilities, furthering their career and their usefulness at their practice.

\section{Legally speaking}

Every person working in radiography for medical and dental purposes are working under the Ionising Radiation Regulations 1999, referred to as IRR99. They are concerned with the protection of workers and patients. A further regulation, the Ionising Radiation (Medical Exposure) Regulations 2000 , takes patient protection further.

Every dental practice using ionising radiation (that is taking $\mathrm{x}$-rays) in the UK must inform The Health and Safety Executive 


\section{"Who is involved" \\ The regulations mentioned in the \\ text say that people involved in radiography in the dental practice must be given specific responsibili- ties depending on their role. Here are the specific responsibilities and duties: \\ The Legal Person is responsible for putting into place all of the requirements of the legislation. This is often the practice owner or one of the partners of the practice. \\ A Radiation Protection Adviser (RPA) provides advice on comply- ing with the requirements of the legislation. This is usually the person or company that provides routine radiation surveys \\ A Radiation Protection Supervisor \\ (HSE) of this. If the practice has a change of ownership or if the practice relocates, then the HSE should be informed of this too. \\ The room or rooms where dental $\mathrm{x}$-rays are taken should have a controlled area around the dental $\mathrm{x}$-ray equipment and nobody should be allowed to enter this area with the exception of the patient (and possi- bly an assistant if the patient is a child or needs help) when the radiograph is being taken. This area should be within 1.5 metres of the $\mathrm{x}$-ray tube and it should not extend beyond the room or surgery. Staff, including the operator should stand outside the con- trolled area during the taking of the radi- ograph. Sometimes a protected area is provided for operators behind a special divide. The operator should be able to see the patient and the $\mathrm{x}$-ray tube warning light while taking radiographs and be able to ensure that nobody comes through the door of the room during exposure.} implements the Local Rules (see section 'legally speaking', above) and can be an appropriately trained dentist or PCD.

\section{A Referrer is a dentist who refers a} patient for a radiograph. A PCD cannot act as a referrer

\section{An IRMER practitioner is a dentist} who takes responsibility for justifying an individual's medical exposure to ensure that the benefit to the patient outweighs the detriment of the exposure.

\section{An Operator is any person who} carries out all or part of the practical aspects of taking a radiograph. This includes: Patient identification: positioning the film, the patient and the $x$-ray tube head; setting the exposure parameters; pressing the exposure button; processing the films; evaluation of quality; exposing test objects as part of a quality programme

Roles that PCDs may undertake are: Radiation Protection Supervisor, and Operator. Anybody undertaking any of these roles should be properly trained.

\section{What if something goes wrong?}

If a patient has received an exposure much greater than intended (around 20 times the intended dose), the Legal person should consult an RPA immediately. If the cause of the overexposure is deemed to be an equipment malfunction, the local HSE should be notified. If the cause of the overexposure is due to clinical or operator error, the IRMER inspectorate should be notified.

\section{Radiation Prołection File}

Every practice should have a Radiation Protection File. This should hold as much information as possible about the procedures that the practice uses to ensure radiation protection. This includes: Local rules and Written procedures for patient protection. It could also contain the information about the training of all members of the practice involved in taking radiographs. The Radiation Protection File should be kept up to date so that it continues to be useful and effective. The file should also include details of the arrangements for maintenance and testing of the $\mathrm{x}$-ray equipment; significant findings of risk assessments; staff instructions; review programmes to keep local rules up to date and the arrangements for investigating and reporting incidents.

\section{Locall Rules}

At the very minimum they should include:

- The Radiation Protection Adviser's details
- Identification of each controlled area and arrangements for restricting access

- Summary of working instructions (eg stand behind protection panel or outside the controlled area)

- Summary of contingency arrangements

- Dose investigation level to decide whether personal monitoring is needed.

Other information that could be included are: the Legal person's details, the Radiation Protection Adviser's contact details, arrangements for personal dosimetry, arrangements for pregnant staff and a reminder to staff to report any faults or if there is an over-exposure.

\section{Written Procedures}

These are required to describe the protocols that are in place for patient protection; such as patient identification, identification of people entitled to act as referrer, IRMER practitioner or operator, ensuring a quality assurance programme is followed, assessment of patient dose, and others.

\section{Training}

Every IRMER practitioner and operator must be adequately trained and undertake continuing education to keep up to date. A training record should be maintained (may be kept in the Radiation Protection File) and be kept available for inspection.

Operators who are a dental nurse and are responsible for setting exposure parameters or positioning the film, patient and tube head, should possess a Certificate in Dental Radiography (see box to the right - how to obtain the NEBDN Certificate in Dental Radiography). If the operator is a Hygienist or Therapist, they should have received an equivalent level of training. This knowledge should be up-dated at least every 5 years.

From 13 May 2005, all dental nurses who take radiographs must have a Certificate in Dental Radiography. Up to this time, all dental nurses who were competently taking dental radiographs prior to 13 May 2000 but had only received the 'Core of Knowledge' training may continue to take radiographs, but need to obtain a Certificate in Dental Radiography to continue taking radiographs after 13 May 2005.

It is preferable that people involved in film processing and quality assurance possess a Certificate in Dental Nursing or NVQ equivalent, or have documentation proving 
The NEBDN Certificate in

Dental Radiography

This comprises a theoretical part and a practical part. A record of experience is maintained as evidence of practical experience and the theory is covered by courses or teaching in practice. There will be an exam to test the theory, and examiners may ask to see the record of experience covering the practical work.

\section{For information on the new qualification contact:}

\section{Jennifer Lavery, NEBDN 110 ,}

London Street, Fleetwood,

LANCS FY7 6EU 01253778417

Jennifer can supply the curriculum and a record of experience.

Other institutions running courses in dental radiography include:

\section{- University of Wales (Bangor) \\ - University of the West of England, Bristol}

\section{- University of Central England}

- Christ Church University College Canterbury

- City University

- University of Derby

- Glasgow Caledonian University

- University of Hertfordshire

- IMECS/ Keele University

- University of Leeds

- Matthew Boulton College, Birmingfham

- University of Portsmouth

- Queen Margaret College

- University of Salford

- University College Suffolk their training for the tasks that they undertake. This training may be provided in-house.

Non-clinical staff who are involved in some of the Operator tasks such as patient identification need to be aware of the need to avoid unnecessary personal exposure.

\section{Protection}

\section{Patient protection}

There is no need for patient protectors such as lead aprons and thyroid collars in routine dental radiographs where modern equipment and techniques ensure that the minimal amount of scatter is directed towards the body. Lead aprons do not protect against internal scatter.

Lead aprons may offer some protection for the vortex occlusal projection and should only be used if the patient is or may be pregnant. Staff members or any other person assisting should wear a lead apron if a handicapped patient or child need assistance during the taking of a radiograph. Lead aprons should be a lead equivalent of at least 0.25 $\mathrm{mm}$ and should not be folded when not in use but stored on hangers and inspected regularly. Thyroid collars should be used when

\section{A training course for an IRMER practitioner or an operator should cover the following topics: \\ - Principles of radiation physics an RPA to anticipate the precautions that are needed to prevent exposure of operators, other staff and other patients. The results should be recorded and then reviewed regu- larly; more frequently if there are changes to practises or the equipment.}

- Risks of ionising radiation

- Radiation doses in dental radiography

- Factors affecting doses in dental radiography

- The principles of radiation protection

- Statutory requirements

- Quality assurance

- Selection criteria (IRMER practitioner only) the thyroid may be in the primary beam.

It is not usually necessary to ask a female patient if she is pregnant, as a dental radiograph does not expose the pelvic area and the dose is small. The risks to the foetus are negligible but patients should be given the option of delaying the radiograph if they wish. If the type of radiograph required means that the pelvic area might be irradiated and the patient is or may be pregnant; consider delaying the radiograph until after delivery. If the radiograph is proceeded with, use a lead apron on the patient and ensure that the foetal dose is minimum.

\section{Staff protection}

If a member of staff regularly takes over 100 intra-oral or 50 panoramic radiographs a week (or the equivalent pro-rata), it is good practice to wear a personal dosimeter. This records the personal dose of radiation over a given time (normally 3 months) and this should be kept on file for at least 2 years. Female employees involved in radiography must be aware of the risks to a foetus should they become pregnant.

\section{Risk assessment}

A risk assessment should be carried out with

\section{Quality assurance}

Quality assurance (QA) is about consistently getting good diagnostic information in a radiograph while exposing patients to the lowest possible radiation dose. Someone in the practice should have responsibility for the QA programme which covers image quality, patient dose, equipment, film processing, training and audits.

The information for this article was taken from the British Dental Association's Advice Sheet A11, "Radiation in Dentistry". Available from the $B D A$ shop.

Radiography and Radiology for Dental Nurses is published in April 2005. Vital will be reviewing this in the Summer issue due out on June 11 th.

Makes sure you don't miss out, turn to page 54 to order a subscription. 


\section{Digital radiography is becoming more commonly used in the dental practice.' You may already have digital radiography operating in your practice or may it not be something that you have yet come across.}

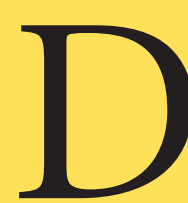

igital radiography originally became available in the dental practice in the 1980s, and although the technical side of digital radiography is very different from conventional radiography, the reasons, justification and referral criteria for a digital $\mathrm{x}$-ray are the same as those for conventional $\mathrm{x}$-ray. ${ }^{1}$

There are many advantages and disadvantages to digital $\mathrm{x}$-ray, some of which are given here. ${ }^{2}$

\section{Advantages}

- Less radiation - As a general rule, the exposure time needed for digital radiography systems is much less than with traditional film.

- Instant images - If a direct sensor systems is being used, the images can appear on the screen immediately. A periapical radiograph on a phosphor plate systems can be developed in about a minute.

- Involving the patient - Digital radiography has the potential to encourage patients' acceptance of and adherence to a treatment plan when they have been able to 'see the problem'.

- Image enhancement and manipulation The contrast and brightness of the monitor being used to view the image can be adjusted, eliminating the need for another exposure. Furthermore, digital $\mathrm{x}$-ray systems come with manipulation software that can be used to enhance the image with colour and 3D and other techniques, all of which will help dentists to diagnose problems - possibly at an earlier stage than would have been possible with film.

- Information sharing - An electronically stored file which could be a word document letter to a specialist or colleague can be quickly sent and viewed by the other party electronically and a second opinion quickly and easily sought. Often more than one person can view the same image at the same time.

- No chemicals - No one on the dental team will be upset about no longer needing to deal with chemicals, processors, missing films or MSDS sheets, none of which are needed with digital radiography.

- Reusable sensors - Because the sensors detecting the $\mathrm{x}$-rays are reusable, there is no need to buy film.

- Time saving - Because the images are available instantly there is much time saved in processing.

- Quick return on investment - Despite the apparently high investment made, the return can easily be realised in less than one year.

\section{Disadvantages}

- Initial capital outlay - The overall cost depends on several factors: choice of direct sensor or phosphor plate system, the number of surgeries to be equipped and whether or not the system will be networked across the practice. In addition, the digital system will need computers with interface cards and further computer screens for patients to see the images.

- Comfort - The average direct sensor is slightly thicker than a traditional x-ray film and as a result, some patients could have trouble tolerating these larger devices. The dentist and the rest of the team need to learn the appropriate way to position the sensor to reduce any patient discomfort.

- Network requirements - As with most new technologies, once the team becomes comfortable with using it, everyone will want access to it. It is possible to just equip one surgery with a digital $\mathrm{x}$-ray system, but having access to the images from several places can be useful. One image can be viewed by a dentist in the surgery at the same time as the same image being accessed from another location in the practice. Although this requires a proper setup of the computer network and daily backup of all data, it adds a powerful dimension to the capabilities. Of course the quality of the monitor that is used to examine the images is important as the exposure time is difficult to determine if the brightness and contrast are not shown correctly. Furthermore, if the computer network goes down, then the images cannot be viewed. Of course all that is needed to view a traditional radiograph is a light box. ${ }^{1}$

- Learning curve - Many practices that purchase digital systems fail to realise the importance of training for the whole team. People who are used to examining traditional film may have difficulty adjusting to seeing images on screen instead. Assisting all staff to feel comfortable with the new system is very important as conversion to a digital system may not be straight forward, and all members of staff would have to learn new procedures.

References

1. Faculty of General Dental Practitioners (UK), The Royal College of Surgeons of England. Pendlebury M E, Horner K, Eaton K A (eds). FGDP (UK) Good Practice Guidelines Selection Criteria for Dental Radiography. London: FGDP (UK) 2004.

\section{2. http://bdhf.atalink.co.uk/articles/} practice-management/131 Accessed 4 Jan 2005 